\title{
Simultaneous Measurement of Rheological Behavior and Conduction Current for an Electro-Rheological Suspension with Cation Exchange Resin Particles
}

\author{
Katsufumi TANAKA, Kensuke IchiZAwa, Ryuichi AKIYAMA, and Atsushi KubONO \\ Department of Polymer Science and Engineering, Kyoto Institute of Technology, \\ Matsugasaki, Kyoto 606-8585, Japan \\ (Received: February 26, 2001)
}

\begin{abstract}
Simultaneous measurement of rheological behavior and conduction current was performed for an electrorheological (ER) suspension with a dilute concentration of cation exchange resin particles of $5 \mathrm{wt} \%$. The stress under the electric field for the ER suspension showed a remarkable dip behavior at higher shear rates: The stress decreased with an increase in the shear rate, and it gradually increased again and approached the stress under no electric field. The apparent conductivity, which was sensitively probed by the conduction current passing through the ER suspension, showed a dip behavior. Further, the apparent conductivity showed a local maximum, which was not found for the ER suspensions with particle concentrations of 10 and $30 \mathrm{wt} \%$.

Key Words : Simultaneous measurement / Electro-rheological suspension / Stress / Conduction current / Apparent conductivity
\end{abstract}

\section{INTRODUCTION}

The electro-rheological (ER) effect is the rheological response of fluids under external modulation of the electric field. That is, reversible changes in the apparent viscosity of some fluids, which are consequent upon the application and removal of the electric field. ${ }^{1)}$ It is known that suspensions composed of polarizable particles and insulating solvents, ${ }^{2-5)}$ and homogeneous fluids such as liquid crystals of small molecules $^{6)}$ as well as macromolecules ${ }^{7)}$ and immiscible polymer blends ${ }^{8}$ show the ER effect. Among these fluids, the ER suspensions are typical and have been widely investigated theoretically and experimentally. The flow curve of a typical ER suspension is generally assumed to be the Bingham flow with the yield stress (dynamic yield stress or induced stress) $\sigma_{y}$ :

$$
\sigma=\sigma_{y}+\eta_{c} \dot{\gamma}
$$

where $\sigma$ is the stress under the electric field, $\eta_{c}$ is the viscosity of the continuous phase of the suspension under no electric field, and $\dot{\gamma}$ is the shear rate. In this model, the yield stress is induced by the external electric field. It is thought that the yield stress is induced by the aggregated structure of polarized particles after application of the electric field. Further, the yield stress at a given strength of the electric field is generally assumed to be constant relative to the shear rate. However, the applicability or limitation of the Bingham flow should be clarified for the purpose of an appropriate design of a system using the ER suspensions in practical application.

The ER effect has been found in our laboratory for the suspensions composed of cation exchange particles and silicone oils with some combinations of the concentration of the particles and the viscosity of the silicone oils. ${ }^{5,9)-12)}$ The particles, the electrical conduction mechanism of which will be the ionic conduction, will show much higher conductivity than the silicone oil. The difference of the conductivity between the particles and a silicone oil would play an important role in the ER effect induced by the DC electric filed.9-12) However, the measurement of the conductivity of only a single particle is extremely difficult. (The ER effect for the suspensions composed of the particles of a polyaniline derivative, which is known as an organic semiconductor, and a silicone oil was also found sufficiently after application of the stepwise electric field. ${ }^{11)}$ )

As reported elsewhere, an ER suspension with a typical concentration of cation exchange resin particles of $30 \mathrm{wt} \%$ and a typical viscosity of silicone oil of $0.02 \mathrm{~Pa}$ s under an electric field of $1 \mathrm{kV} / \mathrm{mm}$ showed the Bingham flow behavior at shear rates up to around $200 \mathrm{~s}^{-1}$. For the ER suspension with the 
same concentration of the particles and with a higher viscosity of silicone oil of $10 \mathrm{Pas}$, the shear-thinning behavior was found. Further, the dip behavior was remarkably found for a dilute ER suspension with a particle concentration of $10 \mathrm{wt} \%$ : The stress decreased as the shear rate increased, and it gradually increased again and approached the stress under no electric field. ${ }^{9)}$ The dip behavior would be induced by the changes in the aggregated structure of polarized particles in the ER suspension. The concentration of the particles will then play an important role in the aggregation of the polarized particles. In a concentrated suspension under an electric field, the aggregated chains of the polarized particles, which connect the electrodes, would further aggregate to form a denser columnar structure. On the other hand, the feature of each single chain of the polarized particles will be emphasized in a dilute ER suspension. The dilute ER suspension would show the dip behavior quite remarkably.

In the present paper, simultaneous measurement of rheological behavior and conduction current was performed for an electro-rheological suspension with a higher viscosity of the continuous phase and a dilute concentration of cation exchange resin particles of $5 \mathrm{wt} \%$. It should be noted that the stress showed a remarkable dip behavior. The apparent conductivity, which was sensitively probed by the conduction current passing through the ER suspension, showed a local minimum corresponding to the dip in the stress. Further, the apparent conductivity showed a local maximum, which was not found so far for the ER suspensions with particle concentrations of 10 and $30 \mathrm{wt} \%$.

\section{EXPERIMENTAL}

Cation exchange resin particles were suspended in a silicone oil. ${ }^{5), 10)}$ In the present study, the concentration of the particles was $5 \mathrm{wt} \%$, and the viscosity of the silicone oil $\left(\eta_{c}\right)$ was $10 \mathrm{~Pa} \mathrm{~s}$.

A rotational rheometer (Rheology Co., Ltd., MR-300V2E) was used $^{10)}$ with a fixture of a coaxial cylinder. Figure 1 shows the schematic illustration of our system for the simultaneous measurement. The DC electric field was applied to the ER suspension, and the strength of the electric field was varied up to $1.0 \mathrm{kV} / \mathrm{mm}$. The stress was calculated from the torque measured with the rheometer using the software provided by the supplier. The shear rate was also calculated from the revolution angle measured with the rheometer as a function of time. The rheometer was calibrated using silicone oils with various viscosities.

In the present experiment, the analog outputs of the torque

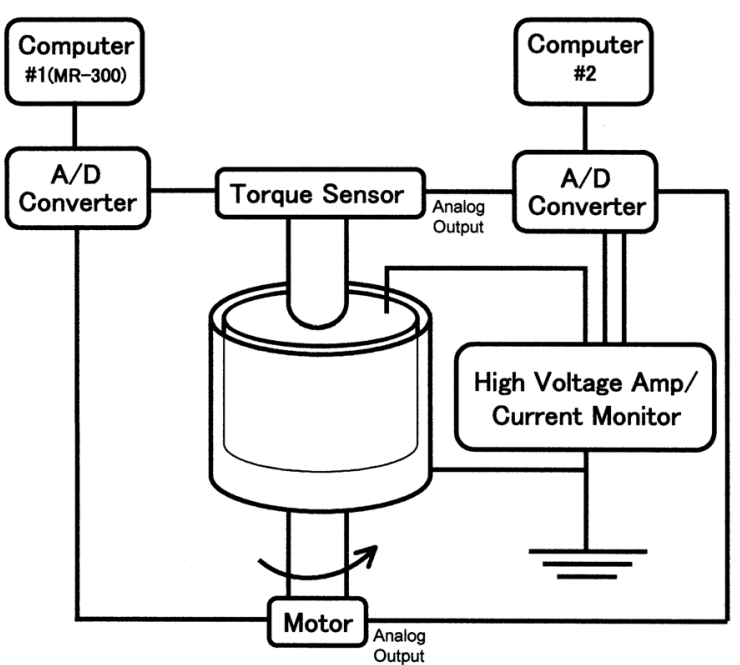

Fig.1 Schematic illustration of our system for the simultaneous measurement.

and the revolution angle from the rheometer were digitized by an analog-to-digital converter, and the data were stored as a function of time in another computer (computer \#2). The current passing through the ER suspension was also digitized and stored in computer \#2. The subtracted current passing through the ER suspension $(\Delta I)$, which was the difference between the current measured under the electric field and that under no electric field, was calculated as a function of time. The subtracted current was averaged over the period of each constant shear rate. The apparent conductivity $\left(\kappa_{a p p}\right)$ was calculated using the averaged subtracted current $(\langle\Delta I\rangle)$ in the following equation:

$$
\kappa_{a p p}=\ln \left(R_{2} / R_{1}\right)(<\Delta I>/ V) / 2 \pi L
$$

where $V$ is the applied voltage, $R_{1}, R_{2}$, and $L$ are, respectively, the inner radius, the outer radius, and the immersed length of the coaxial cylinder of the rheometer. The diameters of the outer cup and the inner bob of the coaxial cylinder were 35.04 $\mathrm{mm}$ and $32.98 \mathrm{~mm}$, respectively. The immersed length was $14.0 \mathrm{~mm}$. In the present experiment, the data for the torque and the revolution angle stored in computer $\# 2$ were used only for determining the period for a constant shear rate.

\section{RESULTS AND DISCUSSION}

\subsection{Dip Behavior of Stress Response}

Figure 2 shows the flow curves of the ER suspension. In the figure, the stress at a given strength of the electric field shows a remarkable dip behavior at higher shear rates: The stress decreases as the shear rate increases, and it gradually increases again and approaches the stress under no electric field. The dip behavior is extremely remarkable for the dilute ER 


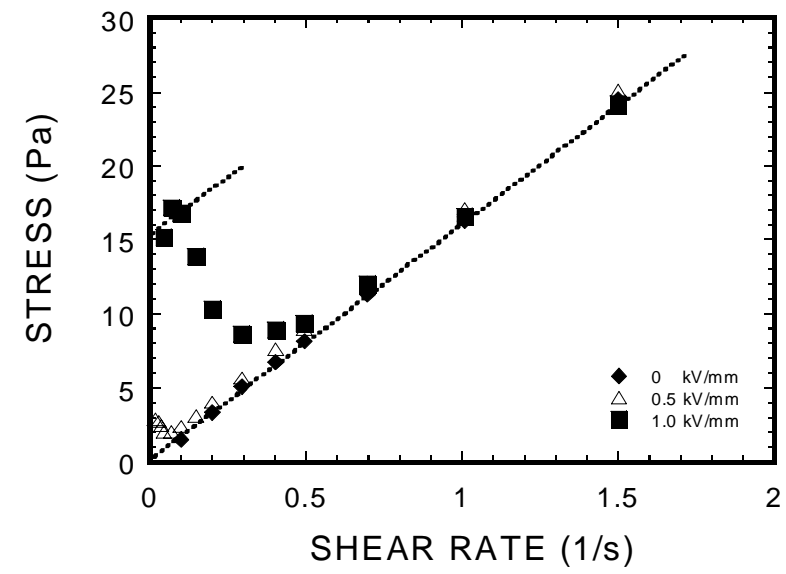

Fig.2 Flow curves of the ER suspension.

suspension.

For discussion in further detail, a general dimensionless parameter called Mason's number, $M_{n}$, is introduced because most important experimental conditions are included in that parameter, representing the ratio of viscous forces to induced dipole interaction forces:

$$
\begin{aligned}
& M_{n}=\eta_{c} \dot{\gamma} / 2 \varepsilon_{\mathrm{c}} \varepsilon_{0}(\beta E)^{2}, \\
& \beta=\left(\kappa_{\mathrm{p}}-\kappa_{\mathrm{c}}\right) /\left(\kappa_{\mathrm{p}}+2 \kappa_{\mathrm{c}}\right),
\end{aligned}
$$

where $E$ is the strength of the electric field, $\varepsilon_{0}$ is the permittivity of free space $\left(8.85 \times 10^{-12} \mathrm{~F} / \mathrm{m}\right), \kappa_{\mathrm{p}}$ is the conductivity of the particles, and $\varepsilon_{\mathrm{c}}$ and $\kappa_{\mathrm{c}}$ are respectively the dielectric constant and the conductivity of the continuous phase of the silicone oil. ${ }^{4}$

In the present study, the conductivity of the particles is assumed to be much higher than that of the silicone oil. Therefore, $\beta$ is assumed to be unity. A typical value of $M_{n}$ for a typical ER suspension is calculated to be 0.08 using $\eta_{\mathrm{c}}=$ $0.02 \mathrm{~Pa} \mathrm{~s}, \dot{\gamma}=200 \mathrm{~s}^{-1}, \varepsilon_{\mathrm{c}}=2.70$, and $E=1 \mathrm{kV} / \mathrm{mm}\left(1 \times 10^{6} \mathrm{~V} /\right.$ $\mathrm{m})$. When an ER suspension with a higher viscosity of the continuous phase and a lower strength of the electric field is investigated, the Mason's number becomes much larger, which corresponds to an experimental condition at higher shear rates. For instance, an important value of $M_{n}=1$ can be obtained for the ER suspension with $\eta_{\mathrm{c}}=10 \mathrm{Pas}, \dot{\gamma}=5 \mathrm{~s}^{-1}, \varepsilon_{\mathrm{c}}=2.76$ and $E$ $=1 \mathrm{kV} / \mathrm{mm}$. The condition corresponds to the shear rate one or two orders higher than the typical case. As shown in Fig.2, the shear rate at the onset of the dip behavior is around $0.1 \mathrm{~s}^{-1}$ at $1.0 \mathrm{kV} / \mathrm{mm}$, which is reduced to $M_{n}=0.02$.

\subsection{Conduction Current Passing through the ER Suspension and Apparent Conductivity under Shear Flow}

Figure 3 shows changes in the conduction current passing through the ER suspension and the shear rate plotted against time. In the simultaneous measurement of rheological behavior and conduction current, the shear rate was decreased step by step from a higher shear rate, and the current as well as the stress (torque) was measured as a function of time. The shear rate was calculated from the time derivative of the revolution angle of the outer cylinder. The current and stress under the electric field were averaged over the period of each constant shear rate. (The shear rate calculated using the data stored in computer \#2 was in good agreement with that stored in our rheometer except for shear rates smaller than $0.1 \mathrm{~s}^{-1}$. A shear rate smaller than $0.1 \mathrm{~s}^{-1}$ was not determined correctly

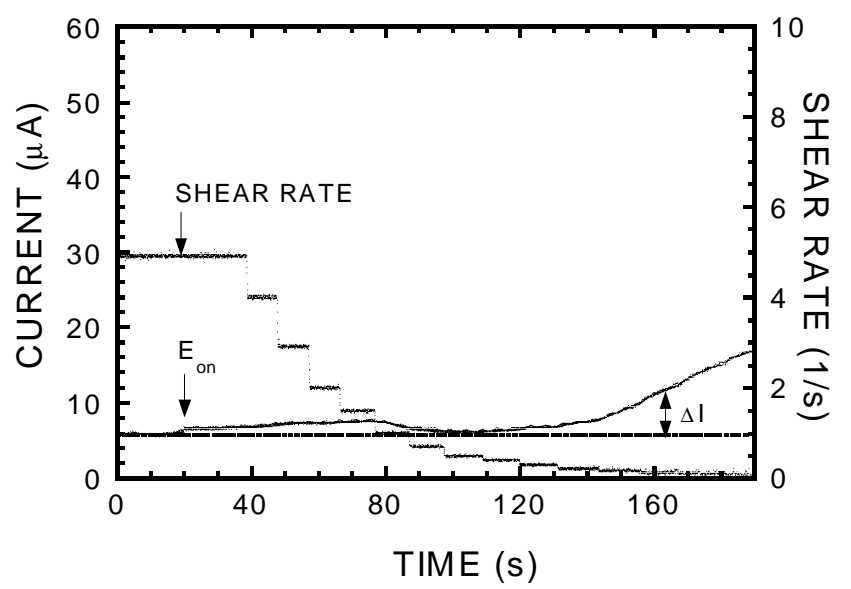

Fig.3 Changes in the conduction current passing through the ER suspension and the shear rate against time. The applied voltage was $1.0 \mathrm{kV}$, and the strength of the electric field was $1.0 \mathrm{kV} / \mathrm{mm}$.

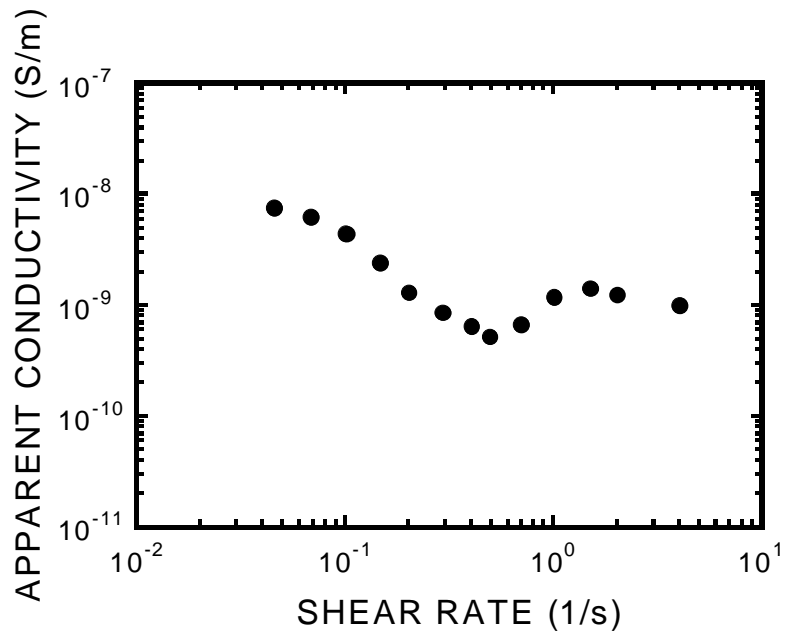

Fig.4 The apparent conductivity of the ER suspension plotted against the shear rate. The applied voltage was $1.0 \mathrm{kV}$, and the strength of the electric field was $1.0 \mathrm{kV} / \mathrm{mm}$. The apparent conductivity was calculated using the data shown in Fig.3.

using computer \#2, because the resolution of the analog-todigital converter and the signal-to-noise ratio were not sufficient for the small signal of the analog output in the 
present experiment. The small stress calculated in computer \#2 was similar. However, the period for a constant shear rate was determined by the shear rate and torque plotted as a function of time.)

In the figure, the shear rate decreases step by step and the conduction current increases to around $80 \mathrm{~s}$. It should be noted that the conduction current after $80 \mathrm{~s}$ decreases and increases again, which corresponds to the dip behavior in the stress response. The complicated behavior of the conduction current against time can be more comprehensible when it is considered as a local minimum and a local maximum in the apparent conductivity relative to the (increasing) shear rate.

Figure 4 shows the apparent conductivity of the ER suspension plotted against the shear rate. In the figure, the apparent conductivity at the lower shear rates decreases as the shear rate increases, which is quite reasonable behavior. That is, the aggregated chains of the polarized particles, which connect the outer cylinder with the inner cylinder as conduction paths (the longer clusters), would be fractured into shorter clusters. The shorter clusters would be recombined and the probability for (or the time required for) the recombination of the shorter clusters becomes smaller (or longer) as the shear rate increases. However, the apparent conductivity shows a local minimum and a local maximum as the shear rate increases, which is closely related to the dip behavior in the stress response. The notable behavior of the apparent conductivity relative to the shear rate is discussed in the next section in comparison with the changes in the reduced stress of the ER suspension plotted against the Mason's number.

\subsection{Apparent Conductivity and Reduced Stress Plotted against the Mason's Number}

Figure 5 shows the apparent conductivity and the reduced stress of the ER suspension plotted against the Mason's number. The stress measured at $1.0 \mathrm{kV} / \mathrm{mm}$ and that measured at $0.5 \mathrm{kV} / \mathrm{mm}$ were reduced by the factor $2 \varepsilon_{\mathrm{c}} \varepsilon_{0}(\beta E)^{2}$, which is proportional to the electric force (with the dimension of stress) acting between the particles. As described in section $3.1, \beta$ is assumed to be unity in the present study. Further, the conductivity measured at $0.5 \mathrm{kV} /$ $\mathrm{mm}$ is not plotted in the figure because the conduction current measured at $0.5 \mathrm{kV} / \mathrm{mm}$ was too small to evaluate the apparent conductivity.

In Fig.5, a master curve is seen for the reduced stress. The Mason's number for the minimum of the reduced stress is around 0.1 . The apparent conductivity also shows a local

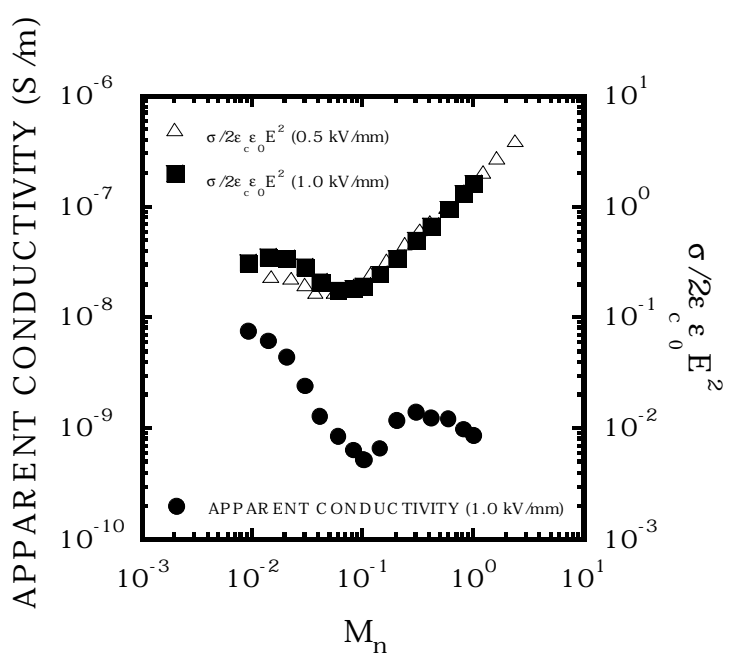

Fig.5 The apparent conductivity and the reduced stress of the ER suspension plotted against the Mason's number. $\left(\eta_{c}=10 \mathrm{~Pa} \mathrm{~s}\right.$, $\varepsilon_{\mathrm{c}}=2.76$ )

minimum around $M_{n}=0.1$, and it shows a local maximum around $M_{n}=0.3$. The apparent conductivity decreases again as the Mason's number increases to 1 .

The local maximum around $M_{n}=0.3$ is unexplained by the macroscopic fracture of the aggregated structure by the shearing force. Around $M_{n}=0.3$, the shorter clusters would be further fractured into much shorter ones (the fractured shorter clusters). Although the particle concentration of the ER suspension investigated in the present study is dilute, the increase in the chance of collision among the polarized particles $^{11,12)}$ and the collision with the electrodes would play an important role in the increase in the apparent conductivity around the Mason's number. The increase in the chance of collision among the polarized particles (or the decreases in the time required for the recombination) would promote the recombination of the fractured shorter clusters in the ER suspension around the Mason's number. Furthermore, the increase in the chance of collision of the polarized particles with the electrodes causes the increase in the conduction current. The local maximum of the apparent conductivity would be caused by both of these factors, although the reasons for the increase in the apparent conductivity are still unclear at the present stage. Above $M_{n}=0.3$, the apparent conductivity decreases again as the Mason's number increases to 1 . In this region, the fractured shorter clusters of the polarized particles would move much more isotropically in the ER suspension. The collisions of the polarized particles with the electrodes would then decrease relatively so that the conduction current decreased in that region. Around $M_{n}=1$, the electric force and the shearing force are comparable, and the aggregation of the polarized particles would be suppressed remarkably in the 
dilute ER suspension.

Finally, the reduced stress above the minimum increases rather continuously, and no behavior corresponding to the local maximum of the apparent conductivity is found in Fig.5 within the limit of experimental error. Therefore, the probe using the conduction current passing through the ER suspension would be effective for the structural changes in the ER suspension under shear flow.

\section{CONCLUSIONS}

In the present study, simultaneous measurement of the conduction current and the rheological behavior was performed for a dilute ER suspension. A remarkable dip behavior at higher shear rates was found for the ER suspension. The conduction current and the apparent conductivity probed sensitively the corresponding behavior of the stress responses. The simultaneous measurement of the rheological behavior and the conduction current is effective for probing the structure changes in the ER suspension under shear flow.

\section{REFERENCES}

1) Jordan TC, Shaw MT, IEEE Trans Electric Insulation, 24, 849 (1989).

2) Filisko FE, Radzilowski LH, J Rheol, 34, 539 (1990).

3) Tao R, Sun JM, Phys Rev Lett, 67, 398 (1991).

4) Tan X, Conrad H, J Appl Phys, 80, 5240 (1996).

5) Tanaka K, Koyama K, Yoshida T, J Soc Rheol, Jpn, 20, 73 (1992).

6) Honda T, Sasada T, Jpn J Appl Phys, 18, 1015 (1977).

7) Tanaka K, Takahashi A, Akiyama R, Phys Rev E, 58, R1234 (1998).

8) Tajiri K, Ohta K, Nagaya T, Orihara H., Ishibashi Y, Doi M, Inoue A, J Rheol, 41, 335 (1997).

9) Tanaka K., T. Takenouchi, R. Akiyama, Proc. of 2000 IEEE International Conference on Industrial Electronics, Control and Instrumentation, (2000), IEEE, pp.1821.

10) Tanaka K. Yamamoto S, Akiyama R, Koyama K, J Soc Rheol, Jpn, 25, 31 (1997).

11) Tanaka K, Nakamura $K$, Takada $K$, Iwaki F, Kubono A, Akiyama R, Kuramoto N, Int J Mod Phys B, 13, 1184 (1999).

12) Tanaka K, Hashimoto $S$, Takenouchi T, Sugimoto I, Kubono A, Akiyama R, Int J Mod Phys B, 15, 930 (2001). 